\title{
VISUALLY EVOKED RESPONSE IN ANESTHETIZED MAN WITH AND WITHOUT INDUCED MUSCLE PARALYSIS ${ }^{*}$
}

\author{
Edward F. Domino and Guenter Corssen \\ Departments of Pharmacology and Anesthesiology \\ University of Michigan Medical Center \\ Ann Arbor, Mich.
}

The use of highly sensitive techniques for recording sensory evoked responses from the human scalp increases the likelihood that the potentials obtained contain electrical components not only from the underlying brain but also from skeletal muscle and other extraneuronal sources. Our concern over the observation of Bickford that the auditory evoked response in man has important skeletal muscle components, prompted us to study the effects of complete skeletal muscle paralysis on the visually evoked response (VER) in lightly anesthetized surgical patients. The effects of thiopental on the VER in man have been reported by Cigánek $(1961 a, b)$. Brazier $(1960 a, b)$ also recorded the averaged visually evoked response in man and animals; she reported the modifications of the visually evoked response in animals by general anesthetics. A study of the effects of a variety of general anesthetics on the VER in man has been recently completed and is being reported elsewhere (Domino et al., 1963).

Inasmuch as light general anesthesia (Stage III, upper plane 1 after Guedel) does not markedly alter the VER in man, and in fact may enhance certain components, it was felt that surgical patients under general anesthesia could be used as subjects to determine the effect of skeletal muscle paralysis on the visually evoked response. Such an approach appeared justified and feasible by selecting patients in whom skeletal muscle paralysis had to be induced for tracheal intubation prior to surgery.

\section{Methods}

Twelve patients of both sexes were selected for this study ranging in age from 9 to 73 years. Generally, patients scheduled for various orthopedic procedures who were in good health and found to be free of any neurological or psychiatrc disorders were utilized. The following equipment and techniques were used for the recording of visually evoked responses: A CAT Model 400 computer was employed to obtain the averaged evoked response. The computer was set for a 500 millisecond analysis with internal trigger at one second intervals. A pulse counter was used to automatically stop after 200 responses. An amplified synchronizing pulse at ordinate 20 was used to trigger a Grass photic stimulator. The flash unit was enclosed in a sound dampened lucite box to reduce the audible lamp click. The flash intensity of the photic stimulator was set at low 2. The lamp was placed six in. away from the subject's eyes.

* This investigation supported in part by grant MY 2653, C4, U.S.P.H.S. 
The patient under study rested comfortably in the supine position on a flat operating room cart. No analgesic narcotics or sedatives were administered for at least 12 hours prior to the recording. The electrodes were placed in accordance with the 10-20 International Electrode System (Jasper, 1958) and electroencephalographic recordings obtained from $\mathrm{Fp}_{1}, \mathrm{~F}_{3}, \mathrm{C}_{3}, \mathrm{P}_{3}, \mathrm{O}_{1}$, and $\mathrm{O}_{\mathrm{z}}$. All scalp electrodes were of the Grass silver disc type. Bentonite electrode paste with a piece of gauze served to anchor the electrode to the scalp. An Offner Type R 8 channel EEG was used for amplification and recording. The filter settings were high 1 and a time constant of 0.3 seconds for recording the EEG. The output voltage of the four channels which recorded $\mathrm{C}_{3}, \mathrm{P}_{3}, \mathrm{O}_{1}$, and $\mathrm{O}_{z}$ were the input to the CAT. The analogue output of the CAT was recorded on one channel (DC input) of the EEG. All EEG recordings were made on the left side of the head to both ears which served as the indifferent site. Throughout this study negativity is represented on the writeout as up. Lead II of the EKG was also recorded.

The following procedure was used throughout: The subject was briefly explained the purpose of the study. An intravenous infusion containing five per cent dextrose in 0.2 per cent saline solution was started. Two sets of VER with the subject's eyes closed were alternated with two sets of VER with eyes open. Subsequently one of the following preanesthetic medications was administered intravenously over a 30 second period: scopolamine $\mathrm{HBr}(0.06 \mathrm{mg} . / \mathrm{kg}$. $)$, atropine sulfate $(0.0092 \mathrm{mg} . / \mathrm{kg}$. $)$, or $1-$ hyoscyamine $\mathrm{HBr}(.0097 \mathrm{mg} . / \mathrm{kg}$.). Three minutes after administration of the preanesthetic medication the sets of light flashes were repeated alternating eyes open and eyes closed. Approximately one-half to two hr. after the preanesthetic medication had been administered general anesthesia was begun. Nitrous oxide-oxygen ( 80 per cent to 20 per cent), halothane (2.0 per cent to 0.5 per cent in oxygen) and methoxyflurane (three per cent to one per cent in oxygen) were administered using a semiclosed circle filter system while cyclopropane-oxygen ( 50 per cent to 10 per cent in oxygen) was administered using a closed circle filter system. In one of the twelve patients it was necessary to induce anesthesia with intravenous thiamylal (three mg./kg.).

When each patient entered surgical anesthesia (Stage III, plane 1) an intravenous infusion of succinylcholine dihydrochloride ( $2 \mathrm{mg} . / \mathrm{cc}$.) diluted in five per cent dextrose and 0.2 per cent saline solution was employed to facilitate the placement of the tracheal tube. Prior to the insertion of the tube the vocal cords were thoroughly sprayed with a two per cent lidocaine solution. Approximately one min. after starting the intravenous succinylcholine drip spontaneous respiration ceased. Adequate respiratory exchange was maintained by manually compressing the breathing bag 12 to 15 times per minute. As soon as a stable anesthetic level of Stage III, upper plane 1 and complete skeletal muscle paralysis was established two sets of light flashes were repeated. Subsequently the intravenous infusion of succinylcholine was stopped and spontaneous respiration restored. A final set of light flashes was presented for comparison of 
the VER with those before and during succinylcholine administration. The patient then was moved into the operating room for the appropriate surgical procedure.

\section{Results}

Physiological characteristics of the averaged visually evoked response. Repetitive light flashes to one or both eyes elicited an average VER in all four channels recorded monopolarly from $\mathrm{C}_{3}, \mathrm{P}_{3}, \mathrm{O}_{1}$ and $\mathrm{O}_{\mathrm{z}}$ to both ears as reference. The earliest events usually were recorded in the occipital areas. Latency and form of the occipital responses, particularly with both eyes closed, appeared similar to those described by Cigánek (1961). Therefore, throughout this report the terminology of Cigánek will be used. Waves three and four were especially prominent in the $\mathrm{O}_{z}-\mathrm{R}$ recording. Recordings from the midline and lateral occipital regions were very similar. Frequently a longer latency negative wave, especially prominent in $\mathrm{C}_{3}-\mathrm{R}$ and less prominent in the $\mathrm{P}_{3}-\mathrm{R}$, was observed. Marked differences were noted in the eyes open and eyes closed responses. Usually the former were of lower voltage and more complex than the latter as described by Van Hof et al. (1962). Inasmuch as the responses obtained with the eyes closed appeared to be less complex, these were used preferentially in this study.

In a series of six unpremedicated normal subjects it was noted that repeated sets of light flashes caused a gradual diminution of the visually evoked response especially as recorded at $\mathrm{O}_{\mathrm{z}}$. Waves three and four appeared to be particularly affected as illustrated in FIGURE 1 . Alteration of the environment such as application of a face mask for administration of either air or oxygen or changing the stimulus to paired light flashes presented a new situation and frequently caused a prompt increase in the amplitude of wave three. It appeared that sensory adaptation constituted a significant problem as would be expected in any study of this type. For this reason a rigorous fixed schedule of stimulus presentations was used as described above.

Effects of methoxyflurane-oxygen anesthesia with and without skeletal muscle paralysis on the VER. Four patients were anesthetized with methoxyflurane-oxygen and complete skeletal muscle paralysis induced with intravenous succinylcholine for tracheal intubation. Prior to the administration of methoxyflurane-oxygen, control VER's were obtained before and after the administration of a muscarinic blocking agent. As illustrated in panel B, FIGURE 2 the intravenous administration of 0.0097 $\mathrm{mg} . / \mathrm{kg}$. of 1-hyoscyamine had relatively little effect on the VER as recorded in all four cortical leads. The patient (F.B.) was a 45-year-old male scheduled for multiple tooth extractions which required the placement of an intratracheal tube via the nose. The patient was anesthetized with methoxyflurane-oxygen and subsequently given succinylcholine by infusion $(2.0 \mathrm{mg} . / \mathrm{cc}$. $)$ to induce skeletal muscle paralysis. Usually a total of 60-120 mg. of succinylcholine was sufficient to produce and maintain 
Trial Number

(FLASH/SEC, 200/TRIAL)

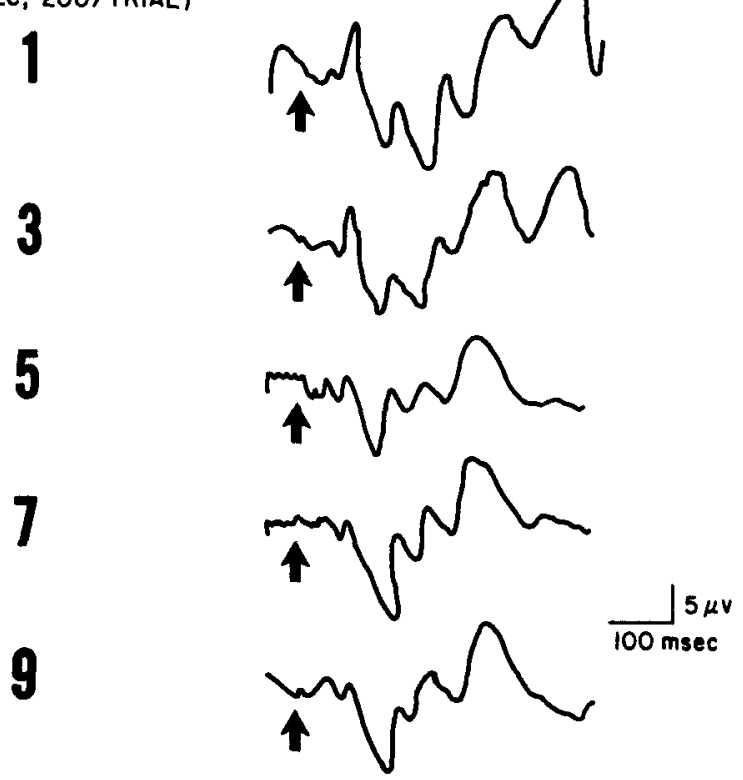

FIGURE 1. Adaptation of visually evoked responses in unanesthetized man. The responses at the inion $\left(\mathrm{O}_{z}\right)$ to both ears as reference $(R)$ for the odd numbered trials with both eyes closed are illustrated. The even numbered trials were with both eyes open. The light stimulus was presented at the arrow every second for 200 flashes. The voltage calibration represents that of the averaged evoked response. Negativity is upward.

skeletal muscle paralysis for seven to ten minutes. After cessation of spontaneous breathing and insertion of the tracheal tube adequate respiratory exchange was maintained by rhythmic manual compression of the breathing bag. Another series of light flashes was presented and the averaged evoked responses were again recorded. These responses are illustrated in FIGURE 2, panel C. A marked diminution of waves one, two, three in the occipital areas and an enhancement of wave four is clearly evident. Also, the latency for these responses appears prolonged and the late negative wave appearing in $\mathrm{C}_{3}$ and $\mathrm{P}_{3}$ was markedly diminished in amplitude. After cessation of the succinylcholine infusion the patient resumed spontaneous respiration. It can be observed that as general anesthesia was continued the visually evoked responses were similar to those without succinylcholine infusion. The EEG indicated at this point that anesthesia had slightly lightened as compared to the anesthetic level during succinycholine administration. Similarly slight differences can be noted in the configurations of the visually evoked responses illustrated in panels $\mathrm{C}$ and D, FIGURE 2.

Another three patients also received methoxyflurane-oxygen anesthesia and their evoked responses were observed with and without neuro- 
muscular paralysis. Similar observations were made as described above. The response from $\mathrm{O}_{2}$ to reference in one of these patients, a 24-year-old male scheduled for an orthopedic procedure on the foot, is illustrated in FiguRE 3. After the administration of $0.0097 \mathrm{mg}$. $/ \mathrm{kg}$. of 1-hyoscyamine a small reduction in the amplitude of wave three and a slight enhancement in the amplitude of wave four was observed. Methoxyflurane-oxygen anesthesia and succinylcholine induced neuromuscular paralysis caused a

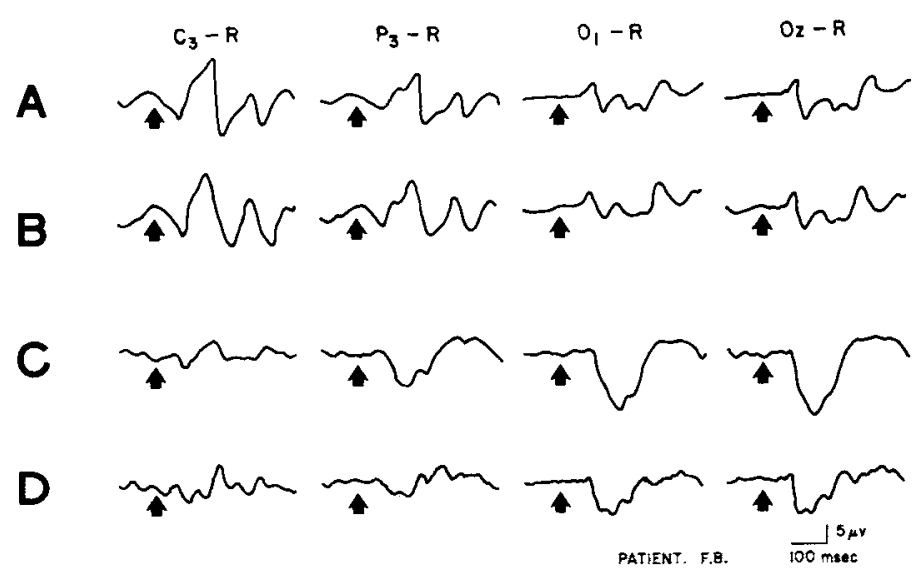

Figure 2. Effects of methoxyflurane-oxygen anesthesia on visually evoked response in man. The responses from four different scalp leads $\mathrm{C}_{3}, \mathrm{P}_{3}, \mathrm{O}_{1}$, and $\mathrm{O}_{2}$ are illustrated. Panel A. Control responses during intravenous infusion of 5.0 per cent dextrose and 0.2 per cent saline; panel B. Responses eight min. after $.0097 \mathrm{mg}$. $/ \mathrm{kg}$. of 1-hyoscyamine hydrobromide given intravenously; panel C. Responses during methoxyflurane-oxygen anesthesia, Stage III, plane 1 with complete skeletal muscle paralysis due to intravenous succinylcholine. Artificial ventilation was maintained manually. The patient had a weak but positive corneal reflex at this time; panel D. Responses during methoxyflurane-oxygen anesthesia, Stage III, plane I following recovery of skeletal muscle function within a few minutes after stopping succinylcholine administration. The patient was not as deeply anesthetized as previously. Note the slight increase in waves 1,2 and 3 and reduction in wave 4 using the terminology of Cigánek. Symbols are similar to those in FIGURE 1.

clear cut enhancement of waves one, two, three, and four. With complete skeletal muscle paralysis present the depth of anesthesia was judged mainly from interpreting the EEG and monitoring cardiovascular activity. The patient's anesthetic depth at this time appeared to be EEG level 1. It can be noted that the evoked responses are especially prominent. When the succinylcholine infusion was stopped the patient resumed spontaneous respiration within five minutes. At this time the depth of anesthesia was established at Stage III, upper plane 1 by slightly increasing the methoxyflurane concentration in the inhaled mixture. Evoked responses were again clearly observed. Thus it can be concluded that VER's particularly recorded from the inion of patients under methoxyflurane-oxygen anesthesia are not markedly altered by skeletal neuromuscular paralysis pro- 
vided that adequate oxygenation and approximately equal levels of anesthesia are maintained.

Effects of halothane-oxygen anesthesia with and without skeletal muscle paralysis on the VER. Four patients were given halothane-oxygen anesthesia and subsequently succinylcholine induced skeletal muscle paralysis was produced for tracheal intubation. The patients were maintained in

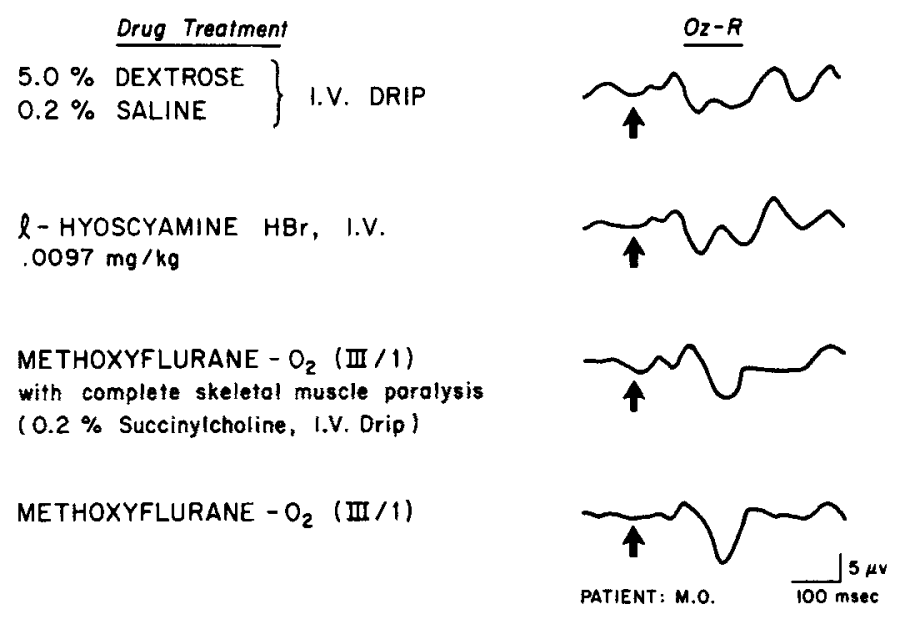

Figure 3. Effects of methoxyflurane-oxygen anesthesia on visually evoked responses in man with and without skeletal muscle paralysis. The recordings at $\mathrm{O}_{\mathrm{z}}-\mathrm{R}$ are illustrated during the various treatments designated. Symbols are similar to those in FIGUREs 1 and 2.

light surgical anesthesia (Stage III, upper plane 1). Figure 4 illustrates the recordings obtained from one of the four patients of this series, a nine year old boy (L.S.) scheduled for removal of a tumor of the left thigh. The control responses from the inion with both eyes closed are typical. After the intravenous administration of $0.006 \mathrm{mg}$. $/ \mathrm{kg}$. of scopolamine $\mathrm{HBr}$ the visually evoked response was reduced slightly. This is particularly evident in wave three and to a lesser extent in wave four. A fusion and delay of waves one, two and three and a reduction in wave four (see FIGURE 4) was observed with the patient maintained in light halothaneoxygen anesthesia, complete skeletal muscle paralysis during the succinylcholine infusion, and adequate artificial respiration. Shortly after cessation of the succinylcholine drip the patient resumed spontaneous respiration and normal skeletal muscle tone recovered. Visually evoked responses during muscle paralysis and after restoration of normal muscle tone were similar. Of special importance was the fact that under light surgical anesthesia wave three was considerably enhanced while somewhat deeper surgical anesthesia resulted in a marked diminution of these responses. It can be concluded that skeletal muscle paralysis with succinylcholine does not markedly affect the visually evoked response recorded from the inion in patients under light halothane-oxygen anesthesia. 
Effects of cyclopropane-oxygen anesthesia with and without skeletal muscle paralysis on the VER. Three patients were anesthetized with a mixture of cyclopropane-oxygen and subsequently were paralyzed with an intravenous succinylcholine infusion for tracheal intubation. As was expected cyclopropane proved to be an extremely potent general anesthetic which, at appropriate concentrations, markedly depressed and even obliterated the VER. This is illustrated in FIGURE 5. Surgical anesthesia (Stage III, plane 2) caused a slow shift in the baseline as illustrated in panels $\mathrm{C}$

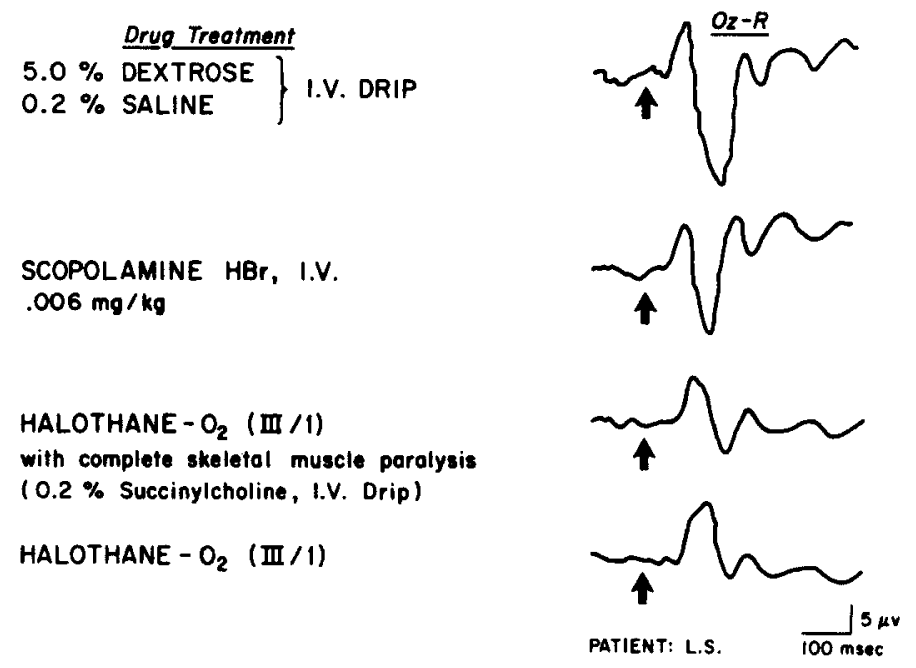

Figure 4. Effects of halothane-oxygen anesthesia on visually eroked response in man with and without skeletal muscle paralysis. The recordings at $\mathrm{O}_{z}-\mathrm{R}$ are illustrated during the various treatments designated. Symbols are similar to those of FIGURES 1 and 2 .

and D, FIGURE 5 . Therefore, it appeared mandatory to maintain the patient in as light surgical anesthesia as possible. Another patient so treated was a 44-year-old female (L.H.) scheduled for a bilateral oophorectomy. The control VER recorded from the inion showed the characteristic configuration as described by Cigánek (FIGURE 6). After the intravenous administration of $0.0092 \mathrm{mg}$. $/ \mathrm{kg}$. of atropine sulfate wave three of the visual evoked response was slightly enhanced. After the induction of cyclopropaneoxygen anesthesia prior to intubation the EEG showed marked delta wave activity indicating level three of cyclopropane. A VER obtained at this time was markedly obliterated similar to the responses to FIGURE 5 . Intravenous succinylcholine infusion $(2 \mathrm{mg}$./ cc.) was begun and skeletal neuromuscular paralysis induced for performing tracheal intubation. When spontaneous respiration ceased and artificial respiration began, the depth of cyclopropane-oxygen anesthesia was lightened until EEG level (1-2) with occasional hypersynchronous 6-9 cps waves was established. During the period of total muscle paralysis the VER was clearly present as de- 


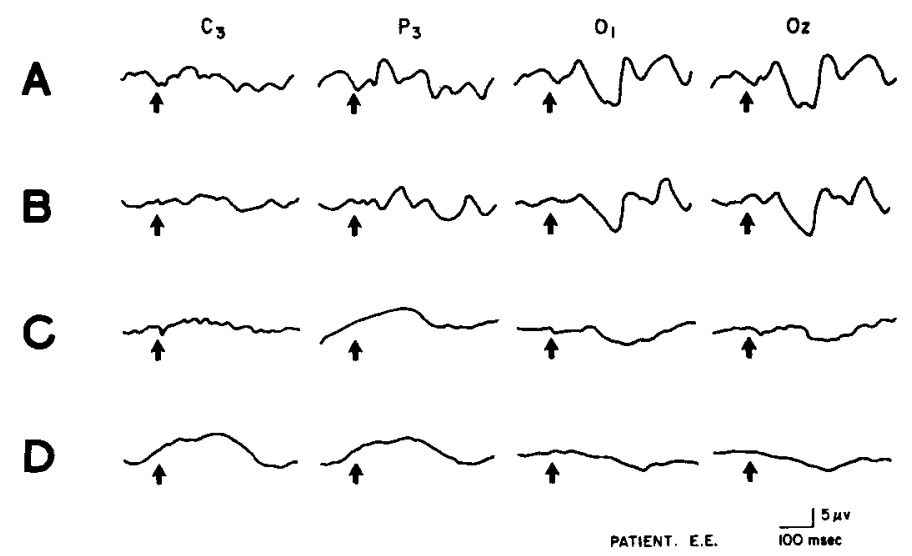

Figure 5. Effects of cyclopropane-oxygen anesthesia on visually evoked response in man. The responses from four different scalp leads $C_{3}, P_{3}, O_{1}$ and $O_{x}$ are illustrated. Panel A. Control responses during intravenous infusion of 5.0 per cent dextrose and 0.2 per cent saline; panel B. Responses $19 \mathrm{~min}$. after $.0092 \mathrm{mg} . / \mathrm{kg}$. of atropine sulfate given intravenously; panel $C$. Responses two min. after cyclopropane-oxygen anesthesia, Stage III, plane 1 to 2 . The lid reflex was negative and the corneal reflex positive; panel D. Responses seven min. after cyclopropane-oxygen anesthesia, Stage III, plane 1 to 2 . The depth of anesthesia was similar to that above. Symbols are similar to those in FIGUREs $l$ and 2.

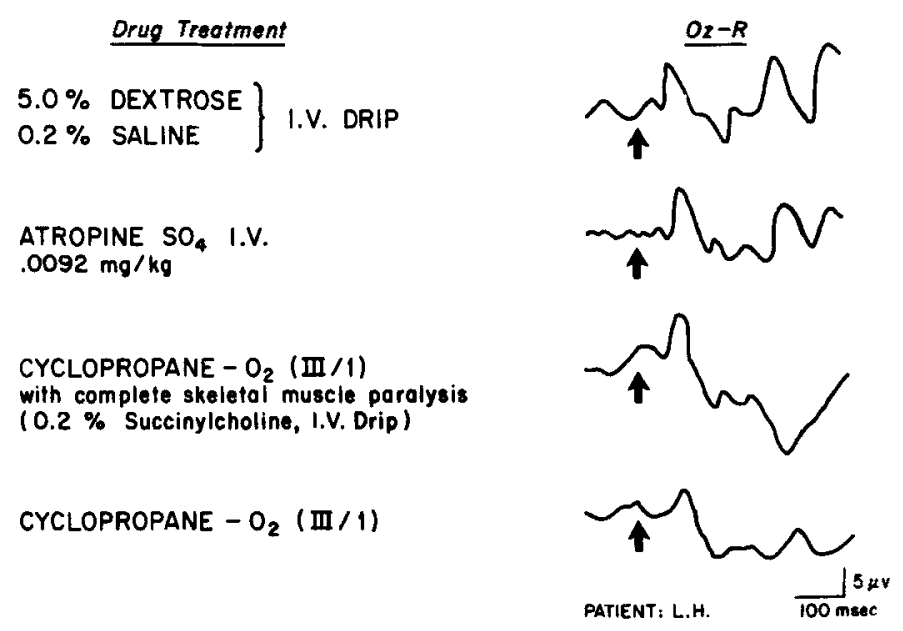

Figure 6. Effects of cyclopropane-oxygen anesthesia on visually evoked response in man with and without skeletal muscle paralysis. The recordings at $\mathrm{O}_{\mathbf{z}}-\mathrm{R}$ are illustrated during the various treatments designated. Symbols are similar to those in FIGURES 1 and 2. 
picted in FIGUPE 6. After stopping the succinylcholine infusion and restoration of neuromuscular function a series of averaged responses to flashing light was repeated with the patient kept at a similar light level of anesthesia. VER's were somewhat similar as shown in FIGURE 6. Another patient (F.D.), a 37-year-old female scheduled for a radical mastectomy, showed similar changes in VER's as indicated in the FIGURE 7. However, in this patient a slightly deeper level of cyclopropane-oxygen anesthesia instantaneously resulted in more reduction of the VER as compared with

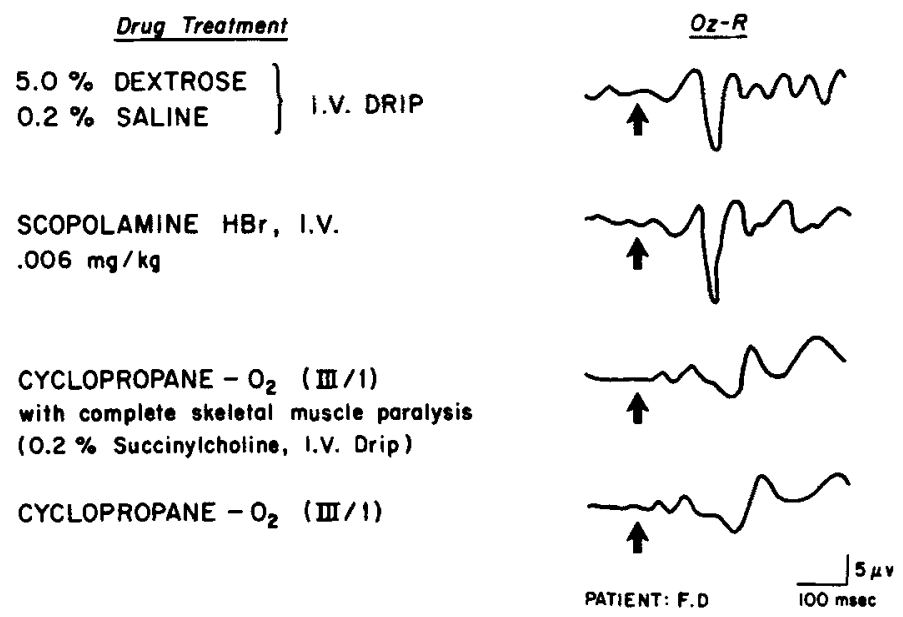

Figure 7. Effects of cyclopropane-oxygen anesthesia on visually evoked response in man with and without skeletal muscle paralysis. The recordings at $\mathrm{O}_{z}-\mathrm{R}$ are illustrated during the various treatments designated. Symbols are similar to those in FIGURES 1 and 2 .

the previous patient. This again indicated the advisability to maintain all patients undergoing cyclopropane-oxygen anesthesia is as light a level of anesthesia as possible. It can be concluded that patients subjected to light cyclopropane anesthesia showed no significant changes in the visual evoked response recorded from the inion during succinylcholine induced skeletal muscle paralysis.

Effects of nitrous oxide-oxygen anesthesia with or without skeletal muscle paralysis on the VER. Two patients were given nitrous oxideoxygen ( 80 per cent-20 per cent) in an attempt to induce surgical anesthesia. In only one patient was it possible to establish Stage III, plane 1 anesthesia. This patient had received scopolamine $(0.006 \mathrm{mg} . / \mathrm{kg}$, intravenously) as a preanesthetic medication. Following induction of anesthesia and succinylcholine induced skeletal muscle paralysis the VER's obtained appeared to be similar to those described previously. Of particular interest is the fact that prior to anesthesia scopolamine caused in this patient markedly diminished waves one, two, and three. These waves were clearly enhanced during nitrous oxide-oxygen anesthesia. Skeletal muscle paralysis with succinylcholine did not markedly affect the VER. As the patient 
recovered from skeletal muscle paralysis following cessation of the succinylcholine infusion the nitrous oxide-oxygen mixture had to be administered by intermittent positive pressure in order to maintain the patient in Stage III. Under these crcumstances wave one, two, and three diminished in amplitude. Nevetheless it can be concluded that succinylcholine induced skeletal muscle paralysis does not markedly modify the VER of a patient lightly anesthetized with nitrous oxide-oxygen.

\section{Discussion}

Surgical patients in whom skeletal muscle paralysis is induced by intravenous succinylcholine to facilitate tracheal intubation may serve as subjects for determining the role of skeletal muscle components in averaged visually evoked responses recorded from scalp electrodes. Of necessity, such procedures must be carried out in patients who are anesthetized to prevent psychic trauma during the period of complete skeletal muscle paralysis. A muscarinic blocking agent had to be given to reduce salivary secretions. Such procedures necessarily introduce certain variables such as drugs, artificial ventilation, and the inability to precisely determine the depth of anesthesia at any phase of the procedure. However, in the course of the study it became apparent that these factors could be reasonably well controlled. Of particular significance was the observation that light surgical anesthesia facilitated certain components of the visually evoked response in man as has been described by Brazier (1961b) in animals.

Surgical patients are naturally apprehensive especially if preanesthetic sedatives are omitted. It may be appropriate to ask to what extent apprehension can modify the visual evoked response prior to surgical anesthesia. Adaptation or "habituation" may be another factor to be considered in modifying the visual evoked response, and thereby interfering with establishing a constant base line. As mentioned above, to study visually evoked responses in unpremedicated unanesthetized subjects to whom a neuromuscular blocking agent is administered would introduce considerable psychic distress which in itself might markedly modify the visual evoked response. Scopolamine premedication enhanced to a certain degree adaptation to the light stimulus perhaps by promoting sleep or reducing anxiety. On the other hand, atropine and 1-hyoscyamine had no effect.

Light surgical anesthesia with any of the agents used resulted in the following alterations of the visual evoked response: (1) prolongation of the latencies of the responses; (2) reduction of the potentials in the nonvisual areas without alteration of the distribution of the potentials, this being especially true of the vertex negative wave which was frequently recorded in the central lead; and (3) enhancement of the amplitude and prolongation of the latency of the evoked responses in the occipital areas, especially waves one, two, and three. This enhancement was quite marked in some individuals.

Increasing the depth of anesthesia frequently caused a reduction of the one, two, three complex and an enhancement of wave four as has been 
reported by Cigánek (1961) for thiopental. The amount of afterdischarge also was considerably reduced by general anesthesia. Skeletal neuromuscular paralysis produced by the intravenous infusion of succinylcholine with the patient's respiratory exchange maintained artificially did not seem to affect markedly the visually evoked response in all four scalp leads studied including $\mathrm{C}_{3}, \mathrm{P}_{3}, \mathrm{O}_{1}$, and $\mathrm{O}_{\mathrm{z}}$. A few of the subjects showed an interesting paradox in that they maintained a slightly positive corneal reflex even though they were completely paralyzed with succinylcholine. This is apparently due to the fact that apposed to the under surface of the levator palpebrae is a layer of smooth muscle (Müller's muscle) which is innervated by the sympathetic nervous system (Adler, 1948). Although all skeletal muscle tone is abolished by succinylcholine, it would be expected that responses of smooth muscle would not be affected. Therefore the seeming paradox is easily resolved. Not all patients showed this phenomenon and therefore it does not appear to detract from our results.

It may be concluded that the visual evoked response in patients under light surgical anesthesia is not altered by succinylcholine induced skeletal muscle blockade. This, of courses, does not prove that there is no muscle component in visually evoked responses of unanesthetized subjects. Nevertheless, the waves described by Cigánek, particularly waves one, two, and three, and four, do not appear to be primarily the result of skeletal muscle components.

\section{Summary}

Twelve surgical patients were used as subjects to determine the effects of skeletal muscle paralysis on the averaged visually evoked response recorded from various scalp electrodes including the central, parietal and occipital areas. While preanesthetic medication with scopolamine tended to reduce wave three of Cigánek and promote adaptation or "habituation," 1-hyoscyamine or atropine had no effect. Light surgical anesthesia (Stage III, upper plane 1) appeared to prolong, enhance and sometimes merge waves one, two, and three. Slightly deeper anesthesia reduced these responses and enhanced wave four. Succinylcholine induced skeletal muscle paralysis with the subjects' ventilation maintained artificially, did not markedly alter the visually evoked responses.

It may be concluded that average visually evoked responses recorded from various areas of the scalp and especially at the inion, are not markedly modified by preanesthetic medication or light surgical anesthesia although the latencies may be prolonged. Under these conditions, skeletal muscle paralysis had no significant effect on the visually evoked response. It would appear that a skeletal muscle component to visually evoked responses in subjects with a normal central nervous system is negligible.

\section{Acknowledgement}

The authors would like to acknowledge the technical assistance of Mary Corcoran in these studies. 


\section{References}

ADLEn, F. H. 1948. Gifford's Textbook of Ophthalmology. P. 156. W. B. Saunders, Co. Philadelphia, $\mathrm{Pa}$.

Brazier, M. A. B. 1960a. Long-persisting electrical traces in the brain of man and their possible relationship to higher nervous activity. EEG Clin. Neurophysiol. Suppl. 13: 347-358.

Brazier, M. A. B. 1960b. Some actions of anesthetics on the nervous system. Federation Proc. 19: 626-628.

CrGÁNEK, L. 1961a. The EEG response (evoked potential) to light stimulus in man. EEG Clin. Neurophysiol. 13: 165-172.

CrgáNEK, L. 1961b. Die elektroencephalographische lichtzeitantwort der menschlichen hirnrinde. Verlag der Slowakischen Akademie der Wissenschatten. Bratislava.

Domino, E. F., G. Conssen \& R. SweEt. 1963. Effects of general anesthetics on the visually evoked response in man. Anesth. and Anal. In press.

JASPER, H. H. 1958. The ten twenty electrode system of the International Federation. EEG Clin. Neurophysiol. 10: 371-375.

Van Hof, M. W., F. VANDER Mark \& W. J. Rietveld. 1962. Influence of formation of retinal image on occipitocortical response to photostimulation. Acta Physiol. Pharmacol. Neerlandica. 10: 317-326. 